\title{
Differential Evolution Solution to Transmission Expansion Planning Problem
}

\author{
Pavlos S. Georgilakis
}

\begin{abstract}
Restructuring and deregulation have exposed the transmission planner to new objectives and uncertainties. As a result, new criteria and approaches are needed for transmission expansion planning (TEP) in deregulated electricity markets. This chapter proposes a new market-based approach for TEP. An improved differential evolution (IDE) model is proposed for the solution of this new market-based TEP problem. The modifications of IDE in comparison to the simple differential evolution method are the following: (1) the scaling factor $F$ is varied randomly within some range, (2) an auxiliary set is employed to enhance the diversity of the population, (3) the newly generated trial vector is compared with the nearest parent, and (4) the simple feasibility rule is used to treat the constraints. Results from the application of the proposed method on the IEEE 30-bus, 57-bus, and 118-bus test systems demonstrate the feasibility and practicality of the proposed IDE for the solution of TEP problem.
\end{abstract}

Keywords Differential evolution - Electricity markets · Power systems · Reference network $\cdot$ Transmission expansion planning

\section{Introduction}

In regulated electricity markets, the transmission expansion planning (TEP) problem consists in minimizing the investment costs in new transmission lines, subject to operational constraints, to meet the power system requirements for a future demand and for a future generation configuration. The TEP problem in regulated electricity markets has been addressed by mathematical optimization as well as by heuristic models (Alguacil et al. 2003; Dechamps and Jamoulle 1980; Latorre et al. 2003; Latorre-Bayona and Pérez-Arriaga 1994; Monticelli et al. 1982; Oliveira

P.S. Georgilakis

National Technical University of Athens (NTUA), GR-15780, Athens, Greece

e-mail: pgeorg@power.ece.ntua.gr 
et al. 1995; Padiyar and Shanbhag 1988; Pereira and Pinto 1985; Romero et al. 2002). Mathematical optimization models for TEP problem include linear programming (Garver 1970; Villasana et al. 1985), dynamic programming (Dusonchet and El-Abiad 1973), nonlinear programming (Youssef and Hackam 1989), mixed integer programming (Alguacil et al. 2003; Bahiense et al. 2001), branch and bound (Haffner et al. 2001), Bender's decomposition (Binato et al. 2001), and hierarchical decomposition (Romero and Monticelli 1994). Heuristic models for the solution of TEP problem include sensitivity analysis (Bennon et al. 1982), simulated annealing (Gallego et al. 1997; Romero et al. 1996), expert systems (Teive et al. 1998), greedy randomized adaptive search procedure (Binato et al. 2000), tabu search (da Silva et al. 2001; Gallego et al. 2000; Wen and Chang 1997), genetic algorithms (GAs) (da Silva et al. 2000; Gallego et al. 1998b), and hybrid heuristic models (Gallego et al. 1998a).

There are two main differences between planning in regulated and deregulated electricity markets from the point of view of the transmission planner: (1) the objectives of TEP in deregulated power systems differ from those of the regulated ones, and (2) the uncertainties in deregulated power systems are much more than in regulated ones.

The main objective of TEP in deregulated power systems is to provide a nondiscriminatory and competitive environment for all stakeholders while maintaining power system reliability. TEP affects the interests of market participants unequally and this should be considered in transmission planning. The TEP problem in deregulated electricity markets has been addressed by probabilistic and stochastic methods (Buygi et al. 2003). Probabilistic methods for the solution of TEP problem include probabilistic reliability criteria method ( $\mathrm{Li}$ et al. 1995), market simulation (Chao et al. 1999), and risk assessment (Buygi et al. 2003, 2004). Stochastic methods for the solution of TEP problem include game theory (Contreras and Wu 2000), fuzzy set theory (Sun and Yu 2000), GA (Georgilakis et al. 2008), and differential evolution (DE) (Georgilakis 2008b).

Nowadays, the TEP problem has become even more challenging because the integration of wind power into power systems often requires new transmission lines to be built (Georgilakis 2008a).

This chapter proposes a general formulation of the transmission expansion problem in deregulated market environment. The main purpose of this formulation is to support decisions regarding regulation, investments, and pricing (Farmer et al. 1995; Kirschen and Strbac 2004; Mutale and Strbac 2000). This chapter proposes an improved differential evolution (IDE) model for the solution of the market-based TEP problem. In particular, the DE algorithm is used to solve the overall TEP problem, whereas in an inner level, that is, for each individual of this evolution-inspired approach, an iterative solution algorithm is required to solve a reference network subproblem.

Evolutionary optimization algorithms have been successfully applied for the solution of difficult power system problems (Georgilakis 2009; Lee and El-Sharkawi 2008). DE is a relatively new evolutionary optimization algorithm (Price et al. 2005; Storn and Price 1997). Many studies demonstrated that DE converges fast 
and is robust, simple in implementation and use, and requires only a few control parameters. In spite of the prominent merits, sometimes DE shows the premature convergence and slowing down of convergence as the region of global optimum is approached. In this chapter, to remedy these defects, some modifications are made to the simple DE. An auxiliary set is employed to increase the diversity of population and to prevent the premature convergence. In the simple DE, the trial vector, or offspring, is compared with the target vector having the same running index, while in this chapter, the trial vector is compared with the nearest parent in the sense of Euclidean distance. Moreover, the comparison scheme is changed according to the convergence characteristics. The scaling factor $F$, which is constant in the original DE, is varied randomly within some specified range. The above modifications form an IDE algorithm, which is applied for the solution of TEP problem. The proposed IDE algorithm is extensively tested on the IEEE 30-bus, 57-bus, and 118-bus test systems, and the results of the proposed IDE are compared with the results of the simple DE (Georgilakis 2008b) as well as with the results obtained by the GA method (Georgilakis et al. 2008).

\section{Problem Formulation}

This section presents a general formulation of market-based TEP problem. The main purpose of this formulation is to support decisions regarding regulation, investments, and pricing (Farmer et al. 1995; Kirschen and Strbac 2004; Mutale and Strbac 2000), and so the main users of this model are regulatory authorities. This formulation is based on the concept of a reference network (Farmer et al. 1995). The determination of such a reference network requires the solution of a type of securityconstrained optimal power flow (OPF) problem (Kirschen and Strbac 2004). A market-based TEP problem that optimizes the line capacities of an existing network has been formulated in Kirschen and Strbac (2004) and Mutale and Strbac (2000). This section extends the work presented in Kirschen and Strbac (2004) and Mutale and Strbac (2000) by formulating a more complex market-based TEP problem that optimizes the topology and the line capacities of a transmission network.

\subsection{Overall TEP Problem}

The objective of the overall TEP problem is to select the new transmission lines that should be added to an existing transmission network (intact system) so as to minimize the overall generation and transmission cost (1), subject to constraints defined by (2)-(9). Alternatively, a different objective also could be considered, such as maximizing the social welfare (de la Torre et al. 2008; Sauma and Oren 2007; Wu et al. 2006). 
The objective function of the overall TEP problem is expressed as follows:

$$
\begin{aligned}
\min \text { AGTIC } & =\min _{w_{b}, P_{p g}, P_{p g}^{c}, T_{b}, T_{b}^{c}, F_{p}^{0}, F_{p}^{c}}\left[\sum_{p=1}^{n p} \tau_{p} \cdot \sum_{g=1}^{n g} C_{g} \cdot P_{p g}\right. \\
& \left.+\sum_{b=1}^{n l} w_{b} \cdot k_{b} \cdot l_{b} \cdot T_{b}\right],
\end{aligned}
$$

where AGTIC (\$) is the annual generation and transmission investment cost, $P_{p g}$ (MW) is the output of generator $g$ during demand period $p, T_{b}(\mathrm{MW})$ is the capacity of transmission line $b, n p$ is the number of demand periods, $\tau_{p}$ is the duration of demand period $p, n g$ is the number of generators, $C_{g}$ is the operating cost of generator $g, n l$ is the number of prospective transmission lines, $k_{b}$ is the annuitized investment cost for transmission line $b$ in $\$ /\left(\mathrm{MW} \mathrm{km}\right.$ year), $l_{b}$ is the length of transmission line $b$ in $\mathrm{km}$, and $w_{b}$ is a binary variable $\left(w_{b}=1\right.$ if line $b$ is built; $w_{b}=0$ if line $b$ is not built).

This optimization is constrained by Kirchhoff's current law, which requires that the total power flowing into a node must be equal to the total power flowing out of the node:

$$
A^{0} \cdot F_{p}^{0}-P_{p}+D_{p}=0, \quad \forall p=1, \ldots, n p,
$$

where $A^{0}$ is the node-branch incidence matrix for the intact system, $F_{p}^{0}$ is the vector of transmission line flows for the intact system during demand period $p, P_{p}$ is the vector of nodal generations for demand period $p$, and $D_{p}$ is the nodal demand vector for period $p$.

The Kirchhoff's voltage law implies the constraint (3) that relates flows and injections:

$$
F_{p}^{0}=H^{0} \cdot\left(P_{p}-D_{p}\right), \quad \forall p=1, \ldots, n p,
$$

where $H^{0}$ is the sensitivity matrix for the intact system.

The thermal constraints on the transmission line flows also have to be satisfied:

$$
-T \leq F_{p}^{0} \leq T, \quad \forall p=1, \ldots, n p
$$

where $T$ is the vector of transmission line capacities.

It should be noted that the constraints (2)-(4) have been derived using a dc power flow formulation neglecting losses.

The constraints (2)-(4) must also be satisfied for contingencies, that is, for credible outages of transmission and generation facilities. As a result, the constraints (5)-(7) also have to be satisfied:

$$
\begin{aligned}
& A^{c} \cdot F_{p}^{c}-P_{p}^{c}+D_{p}=0, \forall p=1, \ldots, n p ; c=1, \ldots, n c, \\
& F_{p}^{c}=H^{c} \cdot\left(P_{p}^{c}-D_{p}\right), \quad \forall p=1, \ldots, n p ; c=1, \ldots, n c, \\
& -T^{c} \leq F_{p}^{c} \leq T^{c}, \quad \forall p=1, \ldots, n p ; c=1, \ldots, n c,
\end{aligned}
$$


where $A^{c}$ is the node-branch incidence matrix for contingency $c, F_{p}^{c}$ is the vector of transmission line flows for contingency $c$ during demand period $p, P_{p}^{c}$ is the vector of nodal generations for demand period $p$ and contingency $c, H^{c}$ is the sensitivity matrix for contingency $c, T^{c}$ is the vector of transmission line capacities for contingency $c$, and $n c$ is the number of contingencies.

The optimization must respect the limits on the output of the generators:

$$
\begin{aligned}
& P^{\min } \leq P_{p} \leq P^{\max }, \quad \forall p=1, \ldots, n p, \\
& P^{\min } \leq P_{p}^{c} \leq P^{\max }, \quad \forall p=1, \ldots, n p ; c=1, \ldots, n c,
\end{aligned}
$$

where $P^{\min }$ is the vector of minimum nodal generations and $P^{\max }$ is the vector of maximum nodal generations.

Since the objective of the optimization is to find the optimal thermal capacity of the lines, these variables can take any positive value:

$$
\begin{aligned}
& T \geq 0, \\
& T^{c} \geq 0 \quad, \quad \forall c=1, \ldots, n c .
\end{aligned}
$$

Network security constraints include generator output constraints and line thermal limits (Kirschen and Strbac 2004; Mutale and Strbac 2000), that is, constraints (4), (7), (8a), and (8b). The solution of the optimization problem of (1)-(3), (5), (6), and (9) provides the capacity for pure transport of each line, $T^{p t}$. On the other hand, the solution of the optimization problem of (1)-(9) provides the optimal capacity of each line, $T^{\text {tot }}$. The capacity for security of each line, $T^{s}$, is defined as $T^{s}=$ $T^{t o t}-T^{p t}$.

\subsection{Reference Network Subproblem}

For a practical power system and for a given number of $n l$ prospective transmission lines, the solution of the overall TEP problem by complete enumeration of prospective transmission network topologies is not realistic, that is why it is proposed to solve the overall TEP problem by DE method, whereas in an inner level, that is, for each individual of this evolution-inspired approach, the reference network subproblem is formulated and solved.

The reference network is topologically identical to an existing (or expanding) transmission network, and the generators and loads are unchanged. The reference network subproblem determines the optimal capacities of transmission lines by minimizing the sum of the annual generation cost and the annuitized investment cost of new transmission lines (10), subject to constraints defined by (2)-(9).

The objective function of the reference network subproblem is expressed as follows (Kirschen and Strbac 2004):

$$
\min \text { AGTIC }^{\mathrm{r}}=\min _{P_{p g}, P_{p g}^{c}, T_{b}, T_{b}^{c}, F_{p}^{0}, F_{p}^{c}}\left[\sum_{p=1}^{n p} \tau_{p} \cdot \sum_{g=1}^{n g} C_{g} \cdot P_{p g}+\sum_{b=1}^{n l^{r}} k_{b} \cdot l_{b} \cdot T_{b}\right],
$$


where $\operatorname{AGTIC}^{\mathrm{r}}(\$)$ is the annual generation and transmission investment cost of the reference network and $n l^{r}$ is the number of prospective transmission lines of the reference network. It should be noted that, for the reference network, it is supposed that all $n l^{r}$ lines are built as well as $0 \leq n l^{r} \leq n l$. It should be also mentioned that for each demand period the reference network subproblem is in fact a type of security-constrained OPF problem (Kirschen and Strbac 2004).

\section{Solution of Reference Network Subproblem}

Because of its size, the reference network subproblem is solved using the iterative algorithm shown in Fig. 1 (Kirschen and Strbac 2004). At the start of each iteration, a generation dispatch is established and the capacity of each line is calculated in such a way that the demand is met during each period and that the transmission constraints are satisfied. Note that at the beginning of the process there are no transmission constraints. The feasibility of this dispatch is then evaluated by performing a power flow analysis for all contingent networks in each demand period (Kirschen and Strbac 2004). If any of the line flows is greater than the proposed capacity of the

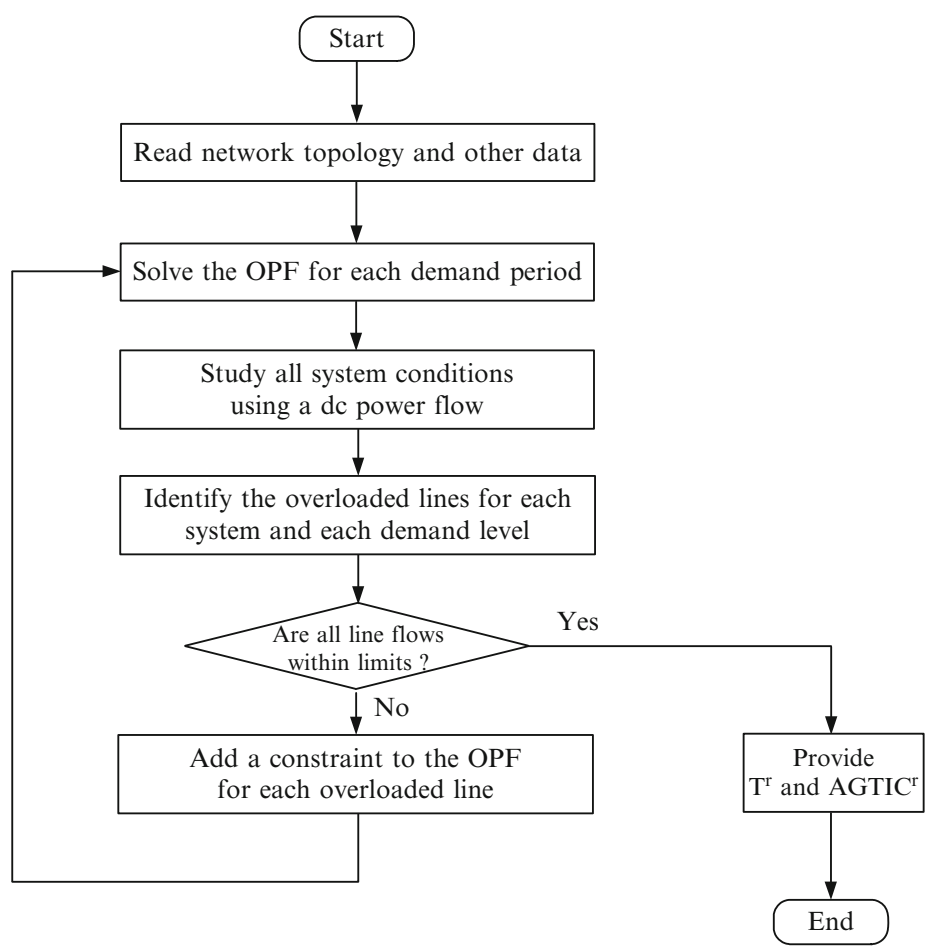

Fig. 1 Flowchart of the algorithm used to solve the reference network subproblem 
line, a constraint is created and inserted in the OPF at the next iteration. This process is repeated until all line overloads are eliminated. At the end, the algorithm provides the optimal capacities $\mathrm{T}^{\mathrm{r}}$ of the transmission lines and the minimum AGTIC $^{\mathrm{r}}$ for the reference network.

\section{Simple Differential Evolution}

The procedure of DE is almost the same as that of the GA, whose main process has selection, crossover, and mutation. The main difference between DE and GA lies in the mutation process. In GA, mutation is caused by the small changes in the genes, whereas in DE, the arithmetic combinations of the selected individuals carry out mutation. An additional difference between DE and GA is the order in which operators are used. It should be noted that DE maintains a population of constant size that consists of $N P$ real-valued vectors $\mathbf{x}_{i}^{G}, i=1,2, \ldots, N P$, where $i$ indicates the index of the individual and $G$ is the generation index. The evolution process of the DE algorithm is as follows.

\subsection{Initialization}

To construct a starting point for the optimization process, the population with $N P$ individuals should be initialized. Usually, the population is initialized by randomly generated individuals within the boundary constraints

$$
x_{j, i}^{0}=\operatorname{rand}_{j, i}[0,1] \cdot\left(x_{j}^{(U)}-x_{j}^{(L)}\right)+x_{j}^{(L)},
$$

where $i=1,2, \ldots, N P, j=1,2, \ldots, D, D$ is the variable dimension, $x_{j}^{(L)}$ and $x_{j}^{(U)}$ are the lower and upper boundary of the $j$ component, respectively, and $\operatorname{rand}_{j, i}[0,1]$ denotes a uniformly distributed random value in the range $[0,1]$.

\subsection{Mutation}

For each target vector, or parent vector $\mathbf{x}_{i}^{G}$, a mutant vector is generated according to

$$
\mathbf{v}_{i}^{G+1}=\mathbf{x}_{n 1}^{G}+F \cdot\left(\mathbf{x}_{n 2}^{G}-\mathbf{x}_{n 3}^{G}\right)
$$

where random indexes $n 1, n 2$, and $n 3$ are integers, mutually different and also chosen to be different from the running index $i$. In the initial DE scheme (Storn and Price 1997), the parameter $F$ is a real and constant factor during the entire optimization process, whose range is $F \in(0,2]$. 


\subsection{Crossover}

The trial vector $\mathbf{u}_{i}^{G+1}$ is generated using the parent and mutated vectors as follows:

$$
\mathbf{u}_{j, i}^{G+1}= \begin{cases}\mathbf{v}_{j, i}^{G+1}, & \text { if } \operatorname{rand}_{j, i}[0,1) \leq C R \text { or } j=k \\ \mathbf{x}_{j, i}^{G}, & \text { otherwise }\end{cases}
$$

where $k \in\{1,2, \ldots, D\}$ is the randomly selected index chosen once for each $i$, and $C R$ is the parameter that is a real-valued crossover factor in the range $[0,1]$ and controls the probability that a trial vector component comes from the randomly chosen, mutated vector $\mathbf{v}_{j, i}^{G+1}$, instead of the current vector $\mathbf{x}_{j, i}^{G}$. If $C R$ is 1 , then the trial vector $\mathbf{u}_{i}^{G+1}$ is the replica of the mutated vector $\mathbf{v}_{i}^{G+1}$.

\subsection{Selection}

To select the population for the next generation, the trial vector $\mathbf{u}_{i}^{G+1}$ and the target vector $\mathbf{x}_{i}^{G}$ are compared, and the individual of the next generation $\mathbf{x}_{i}^{G+1}$ is obtained according to the following rule for minimization problems:

$$
\mathbf{x}_{i}^{G+1}= \begin{cases}\mathbf{u}_{i}^{G+1}, & \text { if } f\left(\mathbf{u}_{i}^{G+1}\right) \leq f\left(\mathbf{x}_{i}^{G}\right) \\ \mathbf{x}_{i}^{G}, & \text { otherwise }\end{cases}
$$

The feature of DE selection scheme is that a trial vector is compared with only one individual, not all the individuals in the current population. Because of the greedy selection scheme, all the individuals of the next generation are as good as or better than their counterparts in the current generation.

\section{Improved Differential Evolution}

This section presents the modifications to the simple DE method that lead to an IDE algorithm.

\subsection{Scaling Factor $F$}

In the initial DE, the scaling factor $F$ in (12) is constant during the optimization process and $F$ takes values in the range $(0,2]$. However, no optimal choice of $F$ has been proposed in the bibliography for DE. All the studies used an empirically derived value, and in most cases $F$ varies from 0.4 to 1 . This means $F$ is strongly problem-dependent and the user should choose $F$ carefully after some trial and error 
tests. In this chapter, $F$ is varied randomly within some specified range, as follows:

$$
F=a+b \cdot \operatorname{rand}_{i}[0,1]
$$

where $a$ and $b$ are positive and real-valued constants, the sum of $a$ and $b$ is less than 1 , and $\operatorname{rand}_{i}[0,1]$ denotes a uniformly distributed random value in the range $[0,1]$.

Consequently, $F$ is different for each generation, and the computation of $F$ by (15) is effective when the optimal value of $F$ is difficult to be determined for complicated problems like TEP.

\subsection{Selection Scheme}

In the original DE, the trial vector or offspring $\mathbf{u}_{i}^{G+1}$ is compared with the target vector $\mathbf{x}_{i}^{G}$, whose index is the same as the running index $i$, using (14). In the modified DE, the trial vector is compared with the nearest target vector in the sense of Euclidean distance. This comparison scheme is employed in the crowding DE algorithm for multimodal function optimization (Thomsen 2004). By this scheme, as the optimization proceeds, the individuals are scattered and gathered around the local optimal points. However, in this chapter, only global optimization is considered, and if there is no improvement of the optimal value during a predefined number of generations, then the comparison scheme is changed to that of the original DE. Therefore, in the initial period of optimization, the DE algorithm explores to find not only global but also local optima, and in the later stage, it searches only for the global optima with greedy selection scheme.

\subsection{Auxiliary Set}

In the selection of the next generation individual, if the trial vector is worse than the target vector, then the trial vector is discarded. To enhance the explorative search and the diversity of the population, an auxiliary set is employed. The auxiliary set $P_{a}$ has the same population size $N P$, and the initialization process is the same as that of the main set, using (11). At each generation, if the trial vector $\mathbf{u}_{i}^{G+1}$ when compared with the corresponding target vector in the main set is found to be worse than its target vector, then the rejected trial vector is compared with the point $\mathbf{w}_{i}^{G}$ with the same running index $i$ in the auxiliary set $P_{a}$. If $f\left(\mathbf{u}_{i}^{G+1}\right)<f\left(\mathbf{w}_{i}^{G}\right)$, then $\mathbf{u}_{i}^{G+1}$ replaces $\mathbf{w}_{i}^{G}$.

To use the solutions in $P_{a}$, after a predefined number of generations, several of the worst solutions in the main set are periodically replaced with the best ones in the auxiliary set by comparing the objective function value. 


\subsection{Treatment of Constraints}

Most optimization problems in the real world have constraints to be satisfied. One common approach to deal with constraints is to penalize constraint violations using an appropriate penalty function (Runarsson and Yao 2000). In this approach, considerable effort is required to tune the penalty coefficients. In this chapter, three selection criteria are used to handle the constraints of the TEP problem:

1. If two solutions are in the feasible region, then the one with the better fitness value is selected.

2. If one solution is feasible and the other is infeasible, then the feasible one is selected.

3. If both solutions are infeasible, then the one with the lowest amount of constraint violation is selected.

It should be noted that the final (best) solution provided by IDE is accepted only if it is feasible; otherwise, the execution of IDE algorithm is repeated.

\subsection{Handling of Integer Variables}

$\mathrm{DE}$ in its initial form is a continuous variables optimization algorithm, and was extended to mixed variables problems (Lampinen and Zelinka 1999). During the evolution process, the integer variable is treated as a real variable, and in evaluating the objective function, the real value is transformed to the nearest integer value as follows:

$$
f=f(\mathrm{Y}) \quad: \quad \mathrm{Y}=y_{j}
$$

where

$$
y_{j}=\left\{\begin{array}{ll}
x_{j}, & \text { if } x_{j} \text { is integer } \\
\operatorname{INT}\left(x_{j}\right), & \text { if } x_{j} \text { is continuous }
\end{array},\right.
$$

where $\operatorname{INT}\left(x_{j}\right)$ function gives the nearest integer to $x_{j}$, and the solution vector is $\mathbf{x}=\left[x_{1}, x_{2}, \ldots, x_{D}\right]$.

\section{Overview of the IDE Solution to TEP Problem}

The IDE algorithm is used to solve the overall TEP problem, whereas in an inner level, that is, for each individual of this evolution-inspired approach, the iterative solution algorithm of Sect. 3 is required to solve the reference network subproblem. In particular, the proposed IDE solution for the market-based TEP problem is composed of the following steps:

1. Given the initial transmission network topology and the planned new generators, create an exhaustive list of candidate new transmission lines. 
2. Create an initial population of candidate solutions. The initial population is randomly created from the exhaustive list of candidate new transmission lines using (11).

3. While the termination criterion is not met, the DE algorithm iterates over the following three phases:

(a) Evaluation of the candidate solutions by solving the reference network subproblem (Sect. 3)

(b) Mutation (with randomly varied scaling factor $F$ ) and crossover

(c) Selection by using the auxiliary set concept

4. As soon as the termination criterion is met (maximum number of generations), the solution proposed by the IDE is the one with minimum operating and investment cost, which simultaneously satisfies all the constraints.

Figure 2 presents the flowchart of the proposed IDE solution to TEP problem.

\section{Results and Discussion}

The proposed IDE algorithm has been extensively tested on the IEEE 30-bus, 57bus, and 118-bus test systems (PSTCA 1999) that are named as case 30, case 57, and case 118, respectively. In particular, case 30 is a modified version of IEEE 30bus system (Alomoush 2000; Buygi et al. 2004). Actual cost data of the Hellenic transmission system have been used in the computations. The results of the proposed IDE have been compared with the results of the simple DE (Georgilakis 2008b) as well as with the results obtained by the GA method (Georgilakis et al. 2008). Pentium 4, 3.20 GHz processor was used in the simulations.

\subsection{Parameter Values for IDE}

The population size and the maximum number of generations are set to 30 and 200 , respectively. The best parameter values for IDE were selected after 100 trials of IDE method with varied values of IDE parameters. The average AGTIC of the final solutions for different values of IDE parameters are shown in Table 1. The best settings are $a=0.4, b=0.4$, and $C R=0.9$, since they provide the minimum AGTIC for case 30 test system, as shown in Table 1. These settings were also confirmed for case 57 and case 118 test systems.

\subsection{Comparison of TEP Methods}

\subsubsection{Case 30}

As can be seen in Fig. 3, the initial transmission network is composed of 32 transmission lines and 28 buses. Bus 11 is a new power plant to be connected to the 


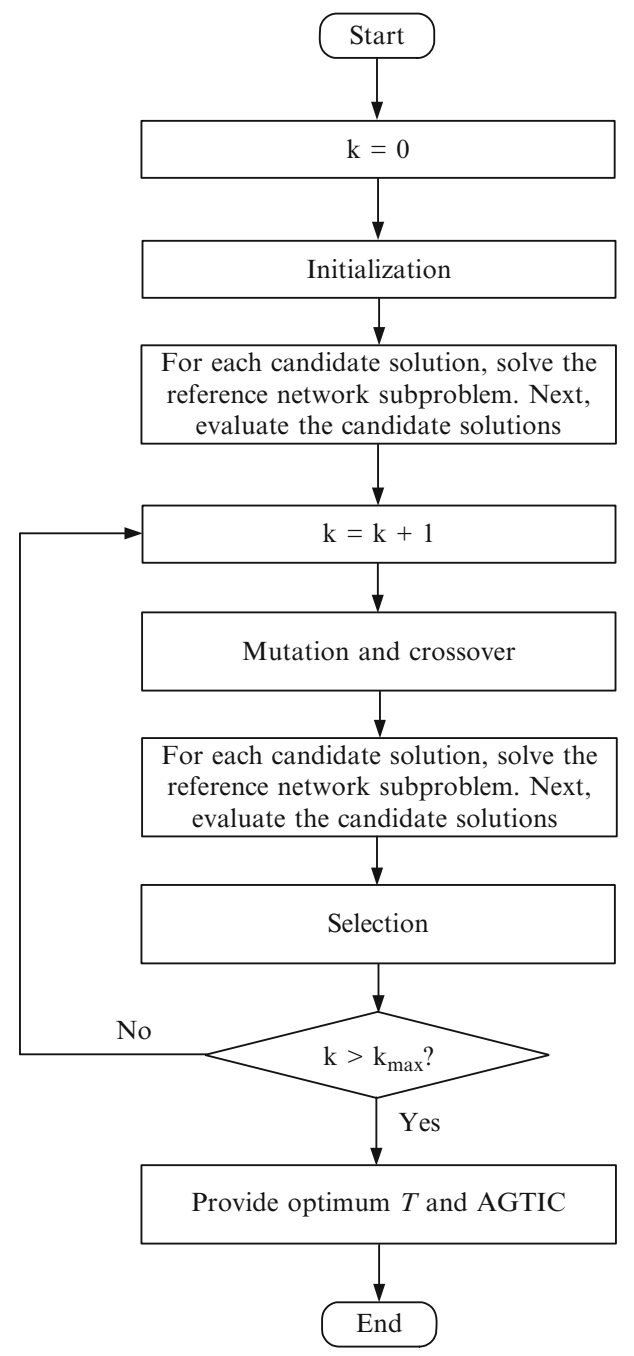

Fig. 2 Flowchart of the proposed improved differential evolution (IDE) solution to transmission expansion planning (TEP) problem

Table 1 Impact of improved differential evolution (IDE) parameters on the computed final solution for case 30 test system

\begin{tabular}{|c|c|c|c|}
\hline \multicolumn{3}{|c|}{ IDE parameters } & \multirow[t]{2}{*}{ AGTIC (M\$) } \\
\hline$a$ & $b$ & $C R$ & \\
\hline 0.2 & 0.3 & 0.8 & 7,203 \\
\hline 0.3 & 0.3 & 0.9 & 7,153 \\
\hline 0.3 & 0.4 & 0.8 & 7,046 \\
\hline 0.4 & 0.4 & 0.9 & 7,043 \\
\hline 0.4 & 0.5 & 0.8 & 7,114 \\
\hline
\end{tabular}




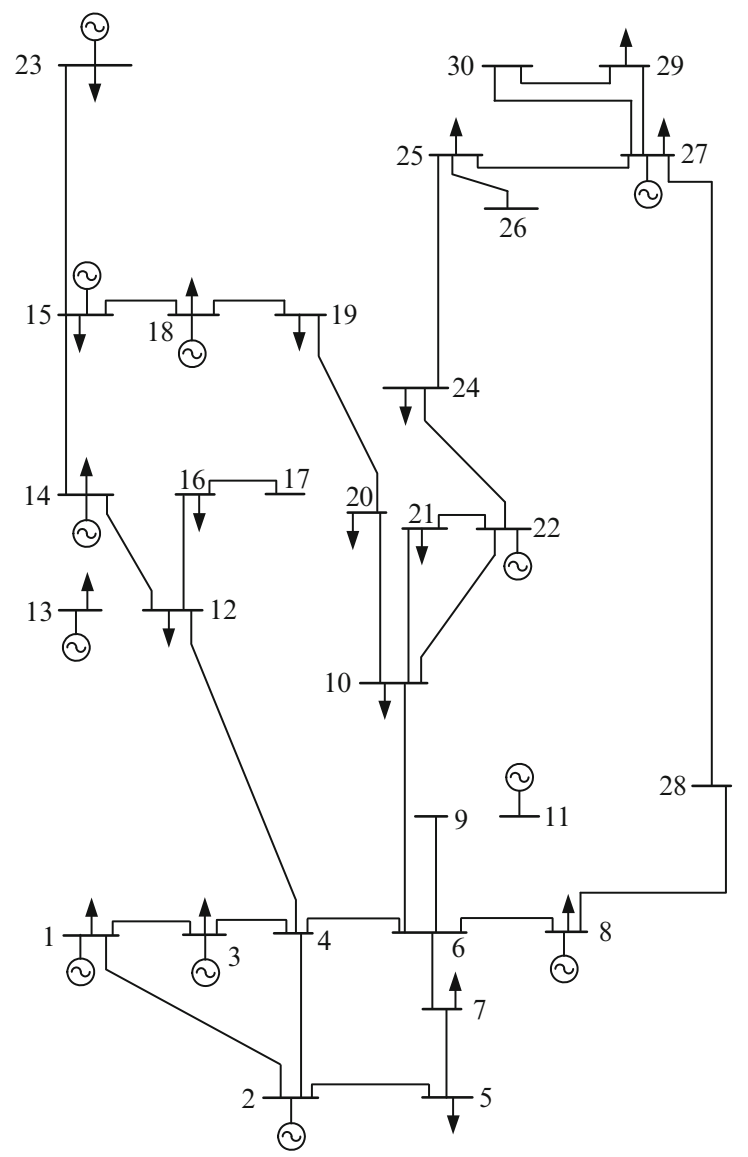

Fig. 3 Single line diagram of the initial transmission network for the modified IEEE 30-bus system

network, and so initially there is no existing transmission line between bus 11 and any bus in the initial network. Bus 13 also corresponds to a new power plant. Table 2 presents the codes of the 32 transmission lines of the initial network of Fig. 3, together with the list of 24 candidate new transmission lines that have been considered for the solution of the transmission expansion problem for the power system in Fig. 3.

The statistic results of the proposed IDE, the simple DE (Georgilakis 2008b), and the GA (Georgilakis et al. 2008) over 100 trials are shown in Table 3. It can be seen in Table 3 that only the proposed IDE technique converges to the best solution, that is, $\$ 7,043$ million minimum AGTIC. The success rate of IDE is $85 \%$, that is, for 85 times out of the 100 trial runs, the same best solution is obtained. It can be seen from Table 3 that the minimum AGTIC provided by the IDE is $1.2 \%$ lower than that obtained by the GA. The application of IDE leads to significant AGTIC savings of $\$ 86$ million in comparison with GA and \$61 million savings in comparison with 
Table 2 Transmission lines of the initial network (Type = I), contingencies of transmission lines of the initial network (Type $=0$ ), and candidate new transmission lines $($ Type $=\mathrm{C}$ )

\begin{tabular}{lllcc|ccccc}
\hline Code & Line & Type & $\begin{array}{c}\text { Reactance } \\
\text { (per unit) }\end{array}$ & $\begin{array}{c}\text { Capacity } \\
\text { (MW) }\end{array}$ & Code & Line & Type & $\begin{array}{c}\text { Reactance } \\
\text { (per unit) }\end{array}$ & $\begin{array}{c}\text { Capacity } \\
\text { (MW) }\end{array}$ \\
\hline 1 & $1-2$ & O & 0.0575 & 250 & 29 & $25-27$ & I & 0.2087 & 15 \\
2 & $1-3$ & I & 0.1652 & 100 & 30 & $27-28$ & O & 0.3960 & 50 \\
3 & $2-4$ & I & 0.1737 & 60 & 31 & $27-29$ & I & 0.4153 & 15 \\
4 & $2-5$ & I & 0.1983 & 100 & 32 & $27-30$ & I & 0.6027 & 15 \\
5 & $3-4$ & I & 0.0379 & 90 & 33 & $2-6$ & C & 0.1763 & 70 \\
6 & $4-6$ & I & 0.0414 & 80 & 34 & $6-28$ & C & 0.0599 & 25 \\
7 & $4-12$ & O & 0.2560 & 50 & 35 & $9-10$ & C & 0.1100 & 30 \\
8 & $5-7$ & I & 0.1160 & 40 & 36 & $9-11$ & C & 0.2080 & 20 \\
9 & $6-7$ & I & 0.0820 & 40 & 37 & $10-17$ & C & 0.0845 & 15 \\
10 & $6-8$ & I & 0.0420 & 40 & 38 & $12-13$ & C & 0.1400 & 15 \\
11 & $6-9$ & I & 0.2080 & 40 & 39 & $12-15$ & C & 0.1304 & 25 \\
12 & $6-10$ & I & 0.5560 & 25 & 40 & $23-24$ & C & 0.2700 & 15 \\
13 & $8-28$ & I & 0.2000 & 10 & 41 & $5-6$ & C & 0.1525 & 50 \\
14 & $10-20$ & I & 0.2090 & 20 & 42 & $6-11$ & C & 0.1982 & 40 \\
15 & $10-21$ & I & 0.0749 & 25 & 43 & $10-11$ & C & 0.1400 & 30 \\
16 & $10-22$ & I & 0.1499 & 15 & 44 & $10-12$ & C & 0.0930 & 20 \\
17 & $12-14$ & I & 0.2559 & 15 & 45 & $10-16$ & C & 0.0940 & 30 \\
18 & $12-16$ & I & 0.1987 & 15 & 46 & $10-28$ & C & 0.0650 & 50 \\
19 & $14-15$ & I & 0.1997 & 15 & 47 & $11-28$ & C & 0.2230 & 25 \\
20 & $15-18$ & I & 0.2185 & 15 & 48 & $12-18$ & C & 0.1400 & 30 \\
21 & $15-23$ & I & 0.2020 & 15 & 49 & $13-14$ & C & 0.2700 & 20 \\
22 & $16-17$ & I & 0.1923 & 15 & 50 & $13-16$ & C & 0.2900 & 20 \\
23 & $18-19$ & I & 0.1292 & 15 & 51 & $15-16$ & C & 0.1800 & 25 \\
24 & $19-20$ & I & 0.0680 & 15 & 52 & $16-18$ & C & 0.1750 & 30 \\
25 & $21-22$ & I & 0.0236 & 15 & 53 & $17-20$ & C & 0.2150 & 20 \\
26 & $22-24$ & I & 0.1790 & 15 & 54 & $19-24$ & C & 0.1560 & 20 \\
27 & $24-25$ & I & 0.3292 & 15 & 55 & $20-24$ & C & 0.1450 & 30 \\
28 & $25-26$ & I & 0.3800 & 15 & 56 & $23-25$ & C & 0.1750 & 30 \\
\hline & & & & & & & & &
\end{tabular}

simple DE. Moreover, both DE methods, the simple DE and the IDE, are faster than the GA method, as Table 3 shows. Consequently, the proposed IDE is very suitable for the solution of the TEP problem.

By applying the proposed IDE method, it has been found that the best-expanded transmission network has selected 7 out of the 24 candidate new transmission lines of Table 2. These 7 transmission lines are shown in Table 4. Figure 4 presents the best-expanded transmission network for the modified IEEE 30-bus system. As can be seen in Fig. 4, the best-expanded transmission network is composed of 39 transmission lines and 30 buses.

Figure 5 presents the capacity for pure transport in each one of the 39 transmission lines of the best-expanded transmission network (Fig. 4) as a percentage of the optimal capacity of the respective transmission line, where the optimal capacity is the sum of two components: (1) the capacity for pure transport and (2) the capacity 
Table 3 Comparison of optimization results for the solution of TEP problem

\begin{tabular}{lccc}
\hline Parameter & \multicolumn{3}{c}{ Method } \\
\cline { 2 - 4 } & GA & DE & IDE \\
\hline Minimum AGTIC (M\$) & 7,129 & 7,104 & $7,043^{\mathrm{a}}$ \\
Minimum AGTIC (\% of minimum AGTIC by GA) & 100.0 & 99.6 & 98.8 \\
Success rate $(\%)$ & 0 & 0 & 85 \\
CPU time (min) & 6.3 & 5.3 & 5.4 \\
CPU time (\% of GA) & 100.0 & 84.1 & 85.7 \\
\hline a7,043 million is considered as the best solution & & & \\
\cline { 3 - 4 } Table 4 New transmission & & Code & Line \\
lines selected by the & & 33 & $2-6$ \\
proposed IDE & & 34 & $6-28$ \\
& & 35 & $9-10$ \\
& & 36 & $9-11$ \\
& & 37 & $10-17$ \\
& & 38 & $12-13$ \\
& & 39 & $12-15$ \\
& &
\end{tabular}

for security. For example, Fig. 5 shows that the transmission line with code 7 , that is, the transmission line between buses 4 and 12 (Table 2), has 38\% capacity for pure transport, while the rest $62 \%$ is its capacity for security. It can be concluded from Fig. 5 that, except for a small number of transmission lines, capacities for pure transport are well below $50 \%$ of the optimal capacities even during the period of maximum demand. This observation confirms the importance of taking security into consideration when solving the transmission expansion problem.

\subsubsection{Case 57 and Case 118}

Figure 6 shows the results obtained by GA, DE, and IDE methods for case 30, case 57, and case 118 test systems. The computing times were 5.4, 21.8, and $87.8 \mathrm{~min}$ for case 30, case 57, and case 118 test systems, respectively. As can be seen in Fig. 6, for the three test systems examined, the IDE method is the best as it provides a TEP solution with minimum AGTIC, which is $0.7-1.2 \%$ lower than the AGTIC of GA and $0.5-0.8 \%$ lower than the AGTIC of DE.

\section{Conclusions}

A general formulation of the transmission expansion problem in deregulated market environment is proposed in this chapter. The main purpose of this formulation is to support decisions regarding regulation, investments, and pricing. This chapter 

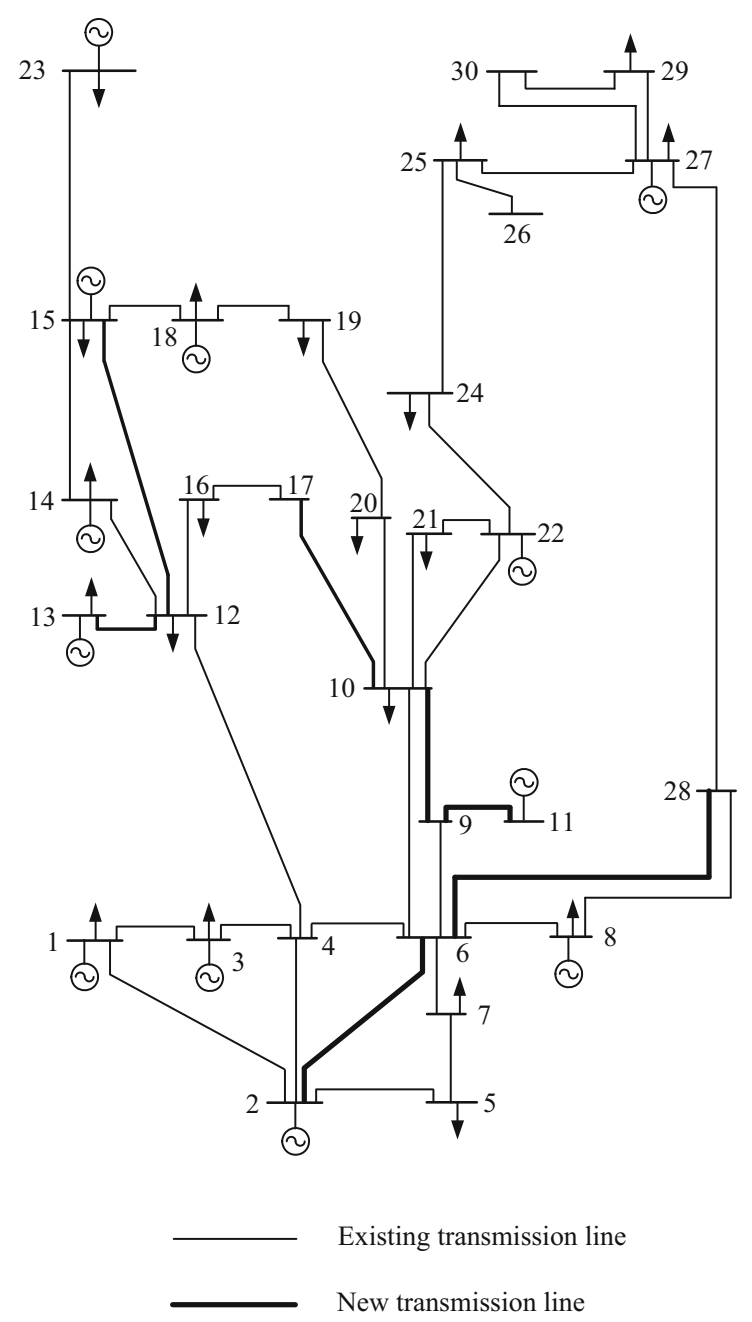

Fig. 4 Single line diagram of the best-expanded transmission network for the modified IEEE 30-bus system

proposes an IDE model for the solution of the market-based TEP problem. The proposed IDE has the following four modifications in comparison to the simple DE: (1) the scaling factor $F$ is varied randomly within some range, (2) an auxiliary set is employed to enhance the diversity of the population, (3) the newly generated trial vector is compared with the nearest parent, and (4) the simple feasibility rule is used to treat the constraints. In particular, the IDE algorithm is used to solve the overall TEP problem, whereas in an inner level, that is, for each individual of this evolutioninspired approach, an iterative solution algorithm is required to solve a reference network subproblem. The proposed method is applied on the IEEE 30-bus, 57-bus, and 118-bus test systems, and the results show that the proposed IDE attains better 


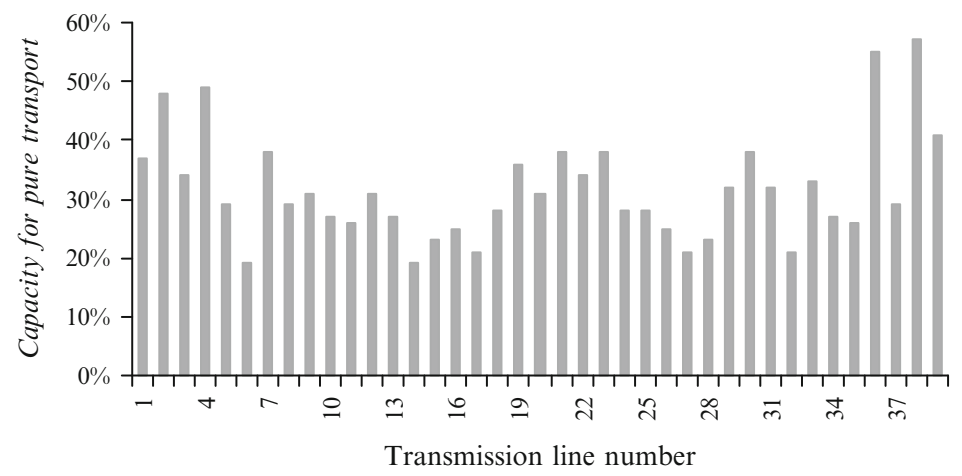

Fig. 5 Capacity needed for pure transport as a percentage of the optimal capacity of each transmission line

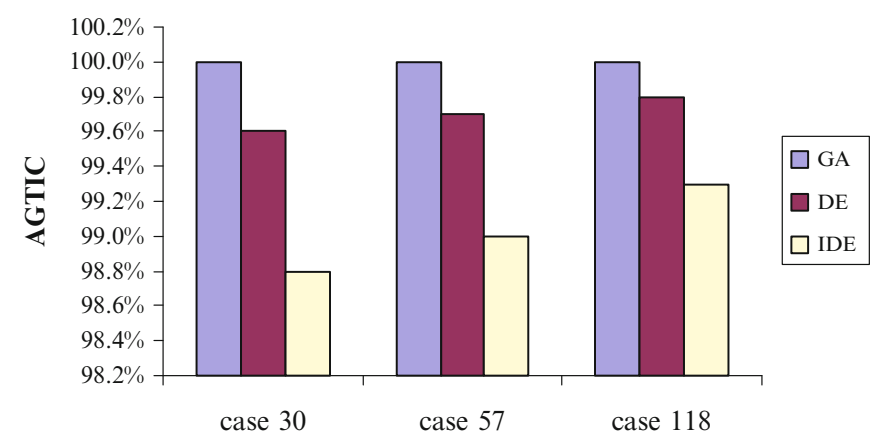

Fig. 6 Annual generation and transmission investment cost (AGTIC) by genetic algorithm (GA), differential evolution (DE), and IDE as a percentage of AGTIC obtained by GA for case 30, case 57, and case 118 test systems

solutions than those found by simple DE and GA. The above four modifications are the possible reasons why IDE outperforms simple DE. Because of its advanced features, IDE also outperforms simple GA. The IDE results show that, except for a small number of transmission lines, capacities for pure transport are well below 50\% of the optimal capacities and this observation confirms the importance of taking security into consideration when solving the transmission expansion problem. A proposal for future work includes comparison of the results obtained by IDE with the results of mixed-integer linear programming formulation of TEP problem (Alguacil et al. 2003; Bahiense et al. 2001; de la Torre et al. 2008).

\section{References}

Alguacil N, Motto AL, Conejo AJ (2003) Transmission expansion planning: a mixed-integer LP approach. IEEE Trans Power Syst 18(3):1070-1077

Alomoush M (2000) Auctionable fixed transmission rights for congestion management. Ph.D. dissertation, Illinois Institute of Technology 
Bahiense L, Oliveira GC, Pereira M, Granville S (2001) A mixed integer disjunctive model for transmission network expansion. IEEE Trans Power Syst 16(3):560-565

Bennon RJ, Juves JA, Meliopoulos AP (1982) Use of sensitivity analysis in automated transmission planning. IEEE Trans Power Apparatus Syst 101(1):53-59

Binato S, de Oliveira GC, de Araújo JL (2000) A greedy randomized adaptive search procedure for transmission expansion planning. IEEE Trans Power Syst 16(2):247-253

Binato S, Pereira MVF, Granville S (2001) A new Benders decomposition approach to solve power transmission network design problems. IEEE Trans Power Syst 16(2):235-240

Buygi MO, Shanechi HM, Balzer G, Shahidehpour M (2003) Transmission planning approaches in restructured power systems. Proc IEEE Bologna Power Tech Conf, Bologna, Italy, June 23-26

Buygi MO, Balzer G, Shanechi HM, Shahidehpour M (2004) Market-based transmission expansion planning. IEEE Trans Power Syst 19(4):2060-2067

Chao XY, Feng XM, Slump DJ (1999) Impact of deregulation on power delivery planning. Proc IEEE Transm Distrib Conf, pp. 340-344, New Orleans, LA, USA, April 11-16

Contreras J, Wu FF (2000) A kernel-oriented algorithm for transmission expansion planning. IEEE Trans Power Syst 15(4):1434-1440

da Silva EL, Gil HA, Areiza JM (2000) Transmission network expansion planning under an improved genetic algorithm. IEEE Trans Power Syst 15(3):1168-1175

da Silva EL, Ortiz JMA, de Oliveira GC, Binato S (2001) Transmission network expansion planning under a tabu search approach. IEEE Trans Power Syst 16(1):62-68

de la Torre S, Conejo AJ, Contreras J (2008) Transmission expansion planning in electricity markets. IEEE Trans Power Syst 23(1):238-248

Dechamps C, Jamoulle E (1980) Interactive computer program for planning the expansion of meshed transmission networks. Electr Power Energy Syst 2(2):103-108

Dusonchet YP, El-Abiad A (1973) Transmission planning using discrete dynamic optimizing. IEEE Trans Power Apparatus Syst 92(4):1358-1371

Farmer ED, Cory BJ, Perera BLPP (1995) Optimal pricing of transmission and distribution services in electricity supply. IEE Proc Generat Transm Distrib 142(1):1-8

Gallego RA, Alves AB, Monticelli A, Romero R (1997) Parallel simulated annealing applied to long term transmission network expansion planning. IEEE Trans Power Syst 12(1):181-188

Gallego RA, Monticelli A, Romero R (1998a) Comparative studies of non-convex optimization methods for transmission network expansion planning. IEEE Trans Power Syst 13(3):822-828

Gallego RA, Monticelli A, Romero R (1998b) Transmission system expansion planning by extended genetic algorithm. IEE Proc Generat Transm Distrib 145(3):329-335

Gallego RA, Romero R, Monticelli AJ (2000) Tabu search algorithm for network synthesis. IEEE Trans Power Syst 15(2):490-495

Garver LL (1970) Transmission network estimation using linear programming. IEEE Trans Power Apparat Syst 89(7):1688-1697

Georgilakis PS (2008a) Technical challenges associated with the integration of wind power into power systems. Renew Sustain Energy Rev 12(3):852-863

Georgilakis PS (2008b) Differential evolution solution to the market-based transmission expansion planning problem. Proc Mediterranean Conf Power Gener Transm Distrib Energy Conversion (MedPower 2008), Thessaloniki, Greece, November 2-5

Georgilakis PS (2009) Spotlight on modern transformer design. Springer, London, UK

Georgilakis PS, Karytsas C, Vernados PG (2008) Genetic algorithm solution to the market-based transmission expansion planning problem. J Optoelectronics Adv Mater 10(5):1120-1125

Haffner S, Monticelli A, Garcia A, Romero R (2001) Specialised branch-and-bound algorithm for transmission network expansion planning. IEE Proc Generat Transm Distrib 148(5):482-488

Kirschen DS, Strbac G (2004) Fundamentals of power system economics. Wiley, Chichester

Lampinen J, Zelinka I (1999) Mixed integer-discrete-continuous optimization by differential evolution, Part 1: the optimization method. Proc 5th Int Conf Soft Computing, pp. 77-81, Brno, Czech Republic, June 9-12

Latorre G, Cruz RD, Areiza JM, Villegas A (2003) Classification of publications and models on transmission expansion planning. IEEE Trans Power Syst 18(2):938-946 
Latorre-Bayona G, Pérez-Arriaga IJ (1994) CHOPIN, a heuristic model for long term transmission expansion planning. IEEE Trans Power Syst 9(4):1886-1894

Lee KY, El-Sharkawi MA (2008) Modern heuristic optimization techniques: theory and applications to power systems. Wiley, Hoboken

Li W, Mansour Y, Korczynski JK, Mills BJ (1995) Application of transmission reliability assessment in probabilistic planning of BC Hydro Vancouver South Metro system. IEEE Trans Power Syst 10(2):964-970

Monticelli A, Santos A Jr, Pereira MVF, Cunha SH, Parker BJ, Praça JCG (1982) Interactive transmission network planning using a least-effort criterion. IEEE Trans Power Apparat Syst 101(10):3909-3925

Mutale J, Strbac G (2000) Transmission network reinforcement versus FACTS: an economic assessment. IEEE Trans Power Syst 15(3):961-967

Oliveira GC, Costa APC, Binato S (1995) Large scale transmission network planning using optimization and heuristic techniques. IEEE Trans Power Syst 10(4):1828-1834

Padiyar KR, Shanbhag RS (1988) Comparison of methods for transmission system expansion using network flow and DC load flow models. Electr Power Energy Syst 10(1):17-24

Pereira MVF, Pinto LMVG (1985) Application of sensitivity analysis of load supplying capability to interactive transmission expansion planning. IEEE Trans Power Apparat Syst 104(2): 381-389

Price KV, Storn RM, Lampinen JA (2005) Differential evolution: a practical approach to global optimization. Springer, Berlin

PSTCA (1999) Power systems test case archive. University of Washington. Available: http://www. ee.washington.edu/research/pstca/

Romero R, Monticelli A (1994) A hierarchical decomposition approach for transmission network expansion planning. IEEE Trans Power Syst 9(1):373-380

Romero R, Gallego RA, Monticelli A (1996) Transmission system expansion planning by simulated annealing. IEEE Trans Power Syst 11(1):364-369

Romero R, Monticelli A, Garcia A, Haffner S (2002) Test systems and mathematical models for transmission network expansion planning. IEE Proc Generat Transm Distrib 149(1):27-36

Runarsson TP, Yao X (2000) Stochastic ranking for constrained evolutionary optimization. IEEE Trans Evol Comput 4(3):284-294

Sauma EE, Oren SS (2007) Economic criteria for planning transmission investment in restructured electricity markets. IEEE Trans Power Syst 22(4):1394-1405

Storn R, Price K (1997) Differential evolution - a simple and efficient heuristic for global optimization over continuous spaces. J Global Optim 11(4):341-359

Sun H, Yu DC (2000) A multiple-objective optimization model of transmission enhancement planning for independent transmission company (ITC). Proc IEEE Power Engineering Society Summer Meeting, pp. 2033-2038, Seattle, WA, USA, July 16-20

Teive RCG, Silva EL, Fonseca LGS (1998) A cooperative expert system for transmission expansion planning of electrical power systems. IEEE Trans Power Syst 13(2):636-642

Thomsen R (2004) Multimodal optimization using crowding-based differential evolution. Proc Evol Comput Conf, pp. 1382-1389, Portland, Oregon, USA, June 20-23

Villasana R, Garver LL, Salon SL (1985) Transmission network planning using linear programming. IEEE Trans Power Apparatus Syst 104(2):349-356

Wen F, Chang CS (1997) Transmission network optimal planning using the tabu search method. Elec Power Syst Res 42(2):153-163

Wu FF, Zheng FL, Wen FS (2006) Transmission investment and expansion planning in a restructured electricity market. Energy 31(6-7):954-966

Youssef HK, Hackam R (1989) New transmission planning model. IEEE Trans Power Syst 4(1): 9-18 